\title{
Aberrant activation of Wnt/catenin signaling and overexpression of ABCG2 contributes to apoptosis down regulation and tumor progression of high grade ovarian cancer
}

\author{
Zhaohua Gui 1,2,3, Hong Yan1,2,3, Jinfeng Wu1,2,3, Mingxun Zhang1,2,3, Airan Wu1,2,3 \\ and Jie $\mathrm{He}^{1,2,3 凶}$
}

1Department of Pathology, The First Affiliated Hospital of USTC, Division of life Sciences and Medicine, University of Science and Technology of China, Hefei, Anhui, 230036, China; ${ }^{2}$ Intelligent Pathology Institute, Division of Life Sciences and Medicine, University of Science and Technology of China, Hefei, Anhui, 230036, China; ${ }^{3}$ Department of Pathology, The First Affiliated Hospital of USTC, Division of life Sciences and Medicine, University of Science and Technology of China, Hefei, Anhui, 230031, China

\begin{abstract}
Side Population (SP) cells are the small pool of CSC like progenitor cells, which are drug resistant and recapitulate tumor generation. The occurrence of SP cells is the major inference for attaining a better treatment and improved patient survival. In this work, we have isolated $6 \%$ SP cells from a high grade ovarian carcinoma. Our functional characterization of SP cells revealed that elevated ABCG2 and anti-apoptotic factors contribute to chemoresistance and increased life span of SP cells. Further, the overexpression of surface antigens, such as CD133 and CD117 in SP cells, are the key driving forces for high clonogenic and invasion properties of SP cells. More importantly, we found by RT-PCR aberrant activation and upregulation of Wnt/ $\beta$-catenin and its downstream targeting genes, such as DKK1 and AXIN2 in SP cells. These findings suggest that development of new anticancer drugs which target Wnt/ $\beta$-catenin signaling might effectively exterminate the SP cells and aid in disease free survival.
\end{abstract}

Keywords: ABCG2; apoptosis; multi-drug resistance; ovary cancer; side population cells; Wnt/ $\beta$-catenin signaling

Received: 13 September, 2020; revised: 23 September, 2020; accepted: 13 October, 2020; available on-line: 26 December, 2020

囚e-mail: gzy1986@163.com

Acknowledgement of Financial Support: This study was supported by The National Natural Science Foundation of China (No. 81702594)

Abbreviations: CSCs, cancer stem cells; EOC, epithelial ovarian cancer; HGSC, high grade serous ovarian carcinoma; FSC, forward scatter; MP, main population; PI, propidium iodide; SP, side population

\section{INTRODUCTION}

Among the gynecological malignancies, epithelial ovarian cancer (EOC) is the primary cause for cancerassociated deaths in women. The occurrence rate of EOC is more than $85 \%$ among the ovarian malignancies and the average survival rate of the patient is about 5 years after diagnosis at metastatic stage (Lengyel, 2010). Standard treatment includes surgery (cytoreductive) and chemotherapy (cisplatin and paclitaxel). Ovarian cancers are categorized as two different types by clinical and pathological approaches: Type I is low grade and heterogenous, whereas as the Type II cancers are high grade and predominantly serous (Kurman et al., 2010; Kurman et al., 2011; He et al., 2014). The high grade serous ovarian carcinoma (HGSC) is a lethal one which has been reported for more than 90\% deaths in women world-wide (Dean et al., 2005; Dalerba et al., 2007). Several studies stated that cancer stem cells (CSCs) or cancer-initiating cells (CICs) are the major obstructions in the treatment regimen, as they cause cancer relapse and chemoresistance (Clevers et al., 2011; Baccelli et al., 2012). The first report on HGSC reported presence of a subtype of ovarian cancer CSCs that are stem-like, very aggressive and become resistant to treatment (He et al., 2014). Subsequently, there are several evidences that the treatment of vain, cancer relapse and progression arise from the existence of sub-population of CSCs or CICs (Dean et al., 2005; Kurman et al., 2010; Kurman et al., 2011). Similarly, research on different cancers also demonstrated that CSCs are able to recreate the tumor with complete heterogeneity and express different surface proteins (CD133, CD44) for self-renewal or differentiation (Bonnet et al., 1997; Dalerba et al., 2007; Clevers et al., 2011). According to the CSC hypothesis, there is a rare set of small progenitor population of cells within the tumor bulk, called Side Population (SP), which repels Hoechst 33342 dye and possesses unique stem like properties (He et al., 2014). The ultimate cause for resistance to anti-cancer drugs is the expression of membrane spanning transporter proteins, such as ABCG2 in SP cells (Vathipadiekal et al., 2012). Further, these purified SP cells are attributed to chemotherapy resistance, tumor regeneration and self-renewal. Therefore, exterminating SP cells is an ultimate modality to provide better treatment strategy and improve the patient survival rate or disease-free state.

One possible and efficient strategy could be achieved by isolating the putative SP (SP) cells and then subjecting them to a functional in depth characterization. In doing so, one such interesting signaling pathway that was identified in CSCs side population cells is $\mathrm{Wnt} / \beta$ catenin. During development, cell migration and differentiation are regulated by Wnt signaling and its downstream targeting proteins. Recent reports in cancer biology described the involvement of Wnt/ $\beta$-catenin signaling in cancer progression and invasion (Song et al., 2010; Taciak et al., 2018 Cheng et al., 2019). Similarly, the abnormal activation of Wnt/ $\beta$-catenin signaling mechanism and its downstream cascades were observed in different cancers, such as endometrioid ovarian, colon, and head and neck squamous cell carcinoma (Dean et al., 2005; Visvader et al., 2008; Song et al., 2010). Therefore, these findings strongly suggest that Wnt/ $\beta$-catenin are one of 
the crucial targets for abolishing CSCs, such as SP cells. Hence, we made an effort to isolate the putative SP cells from epithelial ovarian carcinoma (EOC) and concomitantly monitor the Wnt/ $\beta$-catenin and apoptotic pathways in EOC progression and invasion. Our preliminary findings definitely provide new insights about inactivated pathways for drug response and reveal a different intrinsic mechanistic role of CSCs-mediated tumorigenesis, and pave the way for emerging innovative therapeutic approaches.

\section{MATERIALS AND METHODS}

Cancer sample collection and cell culturing. High grade ovarian carcinoma samples were obtained at the time of surgery (cytoreductive) in accordance with the hospital ethical rules which are approved by The First Affiliated Hospital of USTC, Division of life Sciences and Medicine, University of Science and Technology of China's ethical committee. The approved license number is URY87991. The cancer samples were obtained in agreement with patients' oral consent. As per The First Affiliated Hospital of USTC, Division of life Sciences and Medicine, University of Science and Technology of China's rules and regulations, no data allowing identification of the patients were provided in this manuscript. Patient inclusions: $n=15$; age: $35-45$ ( 7 of them were at 35 and 8 were at 40-45); tumor grade - T4 \& T5; FIGO stage - IIIC and IV, type - recurrence; site - fallopian tube. All samples were assessed by both, the pathological and histological procedures. Cancer tissues were subjected to enzymatic digestion with collagenase III and $0.1 \%$ hyaluronidase (Sigma Aldrich) at $37^{\circ} \mathrm{C}$ for 30 mins. Followed by 2 hour incubation at $37^{\circ} \mathrm{C}$, samples were centrifuge at $1500 \mathrm{RPM}$ to isolate the cells. Subsequently, erythrocytes and other debris were removed by an erythrolysis procedure with $0.2 \% \mathrm{NaCl}$. The isolated cancer cells were washed with Hanks Balanced Solution (HBSS) and filtered through a filter with a pore size of $40 \mathrm{~mm}$ to remove cell clumps. The collected cells were cultured in cell culture dishes (Corining petridishes from Thermoscientific) or T75 flasks with DMEM F-12 (Fisher Scientific) provided with growth factors, such as FGF, EGF, insulin $(5 \mu \mathrm{g} / \mathrm{mL})$ and Bovine Serum Albumin at $0.4 \%$ (Sigma).

Hoechst 33342 dye labelling. After 2-3 passages, the cells were subjected to Hoechst 33342 labeling. Upon confluency, cells were separated from the dishes by washing with $1 \times$ PBS and treated with $1 \mathrm{mM}$ trypsinEDTA (Fishers Scientific). Approximately $10^{6}$ cells $/ \mathrm{mL}$ (counted by hemocytometer) were suspended in DMEM with $10 \%$ FBS and further subjected to labeling with the Hoechst 33342 dye stock (sigma), -bis-benzimide (5 $\mu \mathrm{L} /$ $\mathrm{ml}$ ) alone or in combination with the Verapamil drug (verapamil $-0.8 \mu \mathrm{L} / \mathrm{mL}$ ).

Isolation of SP cells. Two groups were assigned for labeling cells: i) control - cells + Hoechst 33342 dye a; ii) drug treated - cells +Hoechst 33342+Verapamil. Followed by $90 \mathrm{~min}$ staining, cells were spun down at 2000 rpm for 10 minutes at $4^{\circ} \mathrm{C}$ and resuspended in $500 \mu \mathrm{L}$ of HBSS containing $10 \mathrm{mM}$ HEPES. Finally, cells were counterstained with propidium iodide (PI) at $2 \mu \mathrm{g} / \mathrm{mL}$ sample at $4^{\circ} \mathrm{C}$ to exclude the dead cells. This was followed by filtration of cells through a $50 \mu \mathrm{m}$ nylon mesh (BD) to remove cell clumps, and transfer into labeled FACS tubes. Separate tubes with the medium $(10 \%$ DMEM) were kept for sterile sorting of side population $(\mathrm{SP})$ cells and main population (MP) cells.
Flow cytometry analysis. When cells pass through the laser beam, they will scatter the light, which can be detected as a forward and side scatter. Forward scatter (FSC) refers to the cell size and the side scatter (SSC) correlates with the cell density (number of cytoplasmic granules, membrane size). Here, live cell populations were selected against propidium iodide (PI) staining (to exclude the dead cells) which was marked as P1 gated population. From this P1 gated population, side population cells were sorted out using Hoechst 33342, which is a DNA binding dye. Simultaneous monitoring of fluorescence emission by Hoechst 33342 at approximately $450 \mathrm{~nm}$ (SP-Violet) and at $675 \mathrm{~nm}$ (SP-Red) followed by UV excitation, allowed to observe a set of cells that displayed low blue and red fluorescence. These distinct cell populations were gated as $\mathrm{P} 2$, and were found towards the SP-violet region of the dot plot of the FACS profile and are thus called Side Population (SP) cells. The exclusion of Hoechst 33342 by SP cells is an active process involving multi drug resistance transporter 1 (MDR1), a member of the $\mathrm{ABC}$ transporter transmembrane proteins. Experiments were performed in triplicate.

Quantitative real-time PCR primers. RNA extraction was performed with the help of RNAeasy kit from Qiagen as per the manufacturer's protocol. RT-PCR was executed by using an iCycler machine from BioRad. The primers and PCR parameters were employed as previously described (Wamunyokoli et al., 2006; Song et al., 2010). The amplified products were visualized by $2 \%$ agarose gel electrophoresis and the relative mRNA expression levels were adjusted to the GAPDH house keeping gene. The signal intensities were measured using Image-J software and presented as a quantification graph with the help of Pearson's and Spearman's correlation.

Immunostaining. Immunohisto-staining. Cancer tissues were fixed with formalin of $10 \%$ for 24 hours, as per the standard histological procedures. From the paraffin blocks, $4 \mu \mathrm{m}$ serial sections were made and attached to the slides coated with Poly-L-Lysine for immunohistostaining process. These sections were stained with primary antibodies, such as p53 (1:100) and Bcl-2 (1:200) (from Thermofisher), and subjected to overnight incubation. Further procedure was followed as described in (Hill, 2006; Hill et al., 2006). The tissue sections were further viewed by confocal fluorescent microscope and the images were captured at $200 \mathrm{X}$ magnified lens.

Immunocyto-staining. The separated side population (SP) cells and main population (MP) cells were cultured in poly-L-Lysine coated coverslips in 12 well plates (Approximately $10^{5}$ cells). After $24 \mathrm{hrs}$, cells were fixed in 4\% PFA (Para formaldehyde) in $1 \times$ PBS, for $5 \mathrm{~min}$ at $4{ }^{\circ} \mathrm{C}$, followed by blocking with BSA-TBS (1\%) with RNase for $1 \mathrm{hr}$ at room temperature. After PBS wash, FITC conjugated primary antibodies of ABCG2, CD133 and CD117 (diluted 1:100), were added in 1\% BSA-TBS, and the samples were subjected to overnight incubation at $4^{\circ} \mathrm{C}$ and then washed with $1 \times$ PBS for several times to remove the unbound antibodies. Subsequently, the coverslips were mounted on the slides and sealed. The cells were further viewed by confocal fluorescent microscope, FITC was excited at $450 \mathrm{~nm}$ and images were captured at $10 \mathrm{X}$ magnification lens.

Western Blot. For Western blot analysis, the entire procedure was followed as described previously (Song et al., 2010). All primary and secondary horseradish peroxidase (HRP) conjugated antibodies were purchased from Sigma Aldrich, Cell signaling and Santa Cruz Biotechnology Inc. Biorad ECL detection was used to determine 
the protein signals. Biorad protein quantification assay kit was used to evaluate the protein concentration and therefore equal concentration were loaded in all the lanes.

Luciferase assay. In 12 well plate, approximately $10^{6}$ cells were seeded. After 24 hour incubation, transfection of TOPFLASH or FOPFLASH (100 ng), together with pCMV-RL (20 ng), was done with the aid of transfection reagent lipofectamine. After 24 hour incubation, cell lysates were made and the measurement of luciferase activity was performed according to the protocol provided by Promega Dual-Luciferase Reporter Assay System.

Cell transfection. The DKK1 targeting small interfering RNA (siRNA) at $100 \mathrm{nM}$ was mixed with $8 \mu \mathrm{L}$ of RNAiMAX Lipofectamine ${ }^{\circledR}$ transfection reagent (Thermofisher). After 25 minute incubation at room temperature, the reaction mix was directly added to the cells which were subjected to 24-48 hour incubation and then in vitro cell culture assays were performed. The DKK1 RNAi sequence used in this study is: ACU CGG UUC UCA AUU CCA ACG U, obtained from Thermofisher. We have also used Silencer ${ }^{\mathrm{TM}}$ GAPDH siRNA (human) and Silencer ${ }^{\mathrm{TM}}$ Negative Control No. 1 siRNA (from Thermofisher Scientific), as a positive and negative control for RNAi transfection, respectively.

Soft agar assay. In a 6 well plate, the bottom surface was covered with $2 \mathrm{~mL}$ of agar mixture made of: $\mathrm{DMEM} / \mathrm{F} 12+10 \%$ FBS $+0.6 \%$ agar. Once solidified, $2 \mathrm{~mL}$ of agar medium which was made of DMEM/ $\mathrm{F} 12+10 \% \mathrm{FBS}+0.3 \%$ agar and containing $2 \times 10^{4}$ cells were poured into the wells, and allowed to incubate for 3 weeks at $37^{\circ} \mathrm{C}$, followed by crystal violet $(0.005 \%)$ staining for counting the colonies formed.

Cell resistance assay. In 96 well plates, nearly $10^{4}$ cells were seeded and incubate for 24 hours. Subsequently, cells were treated with $10 \mu \mathrm{g} / \mathrm{mL}$ concentration of 5-fluorouracil (5-FU) and incubated for 24, 48 and 72 hours. Prior to the 3 hours of optical density measurement, CCK-8 solution $(10 \mu \mathrm{l})$ was added to wells. The cell resistance and survival rate at different hours of incubation were calculated according to the formula mentioned in (He et al., 2014).

Cell culture assays. We followed the protocol previously described in the literature (Ho et al., 2007) for performing in vitro cell proliferation and matrigel invasion assays.

In vitro cell proliferation assay. Approximately $10^{6}$ cells were seeded in a 96-well plate (in triplicates) and the cell proliferation rate was estimated for 7 consecutive days. 2-3 Hours before optical density measurement, cells were supplemented with CCK-8 solution (10 $\mu \mathrm{L})$ and incubated in $\mathrm{CO}_{2}$ incubator. Consequently, optical density (OD) at $490 \mathrm{~nm}$ was measured and estimated growth rate was presented as a quantification graph.

Matrigel invasion assay. BD six-well matrigel invasion chambers were used for cell invasion measurement (BD Biosciences). Approximately $10^{6}$ cells were seeded per insert in the serum free medium and their outlets were supplemented with DMEM containing 10\% FBS as a chemoattractant. Cells were incubated at $37^{\circ} \mathrm{C}$ for 48 hours and consequently the non-invading/immobile cells were washed away by swabbing top layer of Matrigel with a Q-tip. The membrane containing invading cells was subjected to hematoxylin staining, incubated for $5 \mathrm{~min}$, then washed and mounted on slides. The membrane was further viewed under light microscope at $40 \times$ objective lens and the number of invading cells was counted. The values presented in the graph are the average value of three independent experiments.
Statistical analysis. The values presented in the quantification graph are mean \pm S.D. We have performed Student's t-test to compare the significant differences between two groups and the values defined as significant were $* P<0.05$ and $* * P<0.01$.

\section{RESULTS}

\section{Apoptosis evaluation in ovarian cancer tissues}

The surgically acquired high grade serous ovarian cancer tissues and the analogous normal tissues were examined for the evaluation of apoptosis. First, we have employed the Tunel assay to identify the accumulation of $\mathrm{p} 53$ (tumor suppressor protein) to evaluate the rate of apoptosis in ovarian cancer tissues. We observed that p53 immunostaining is extremely positive in ovarian cancer tissues and the dye is predominantly accumulated in the nuclear region (Fig. 1). Similarly, the immunohistochemistry of Bcl-2 (an anti-apoptotic factor) indicates that it is significantly overexpressed in the ovarian cancer tissues and it showed mostly cytoplasmic staining rather than in the nuclear region (Fig. 1). However, these features were not observed in the control ovary tissues. Therefore, apoptosis downregulation in ovarian cancer is due to the increased expression of anti-apoptotic factor

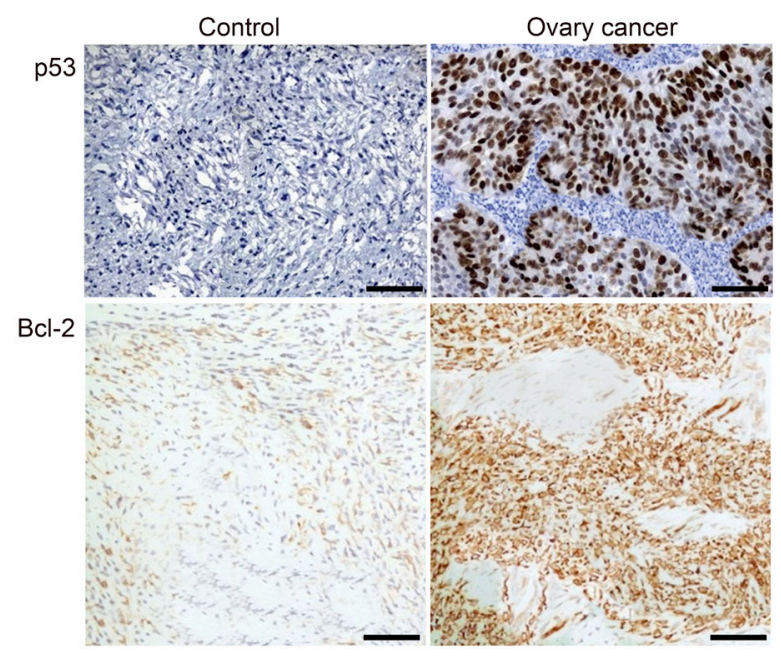

Figure 1. Immunohistostaining pattern of p53 and $\mathrm{Bcl}-2$.

Ovarian cancer tissues show strong p53 staining in almost all cancer nuclei when compared with control tissues. Similarly, the Bcl2 is intensively expressed in ovarian cancer tissue at metastatic stage. Image captured by $200 \times$ magnification and scale bar represents $50 \mu \mathrm{M}$.

bcl-2 and thus ultimately involved in rapid cancer cell proliferation and metastasis.

\section{ABCG2 enrichment and apoptosis downregulation in ovarian cancer SP cells}

It has been proven that SP cells can be sorted by FACs mediated Hoechst 33342 dye efflux method. In all the ovarian cancer samples, we have identified and sorted $6 \%$ of SP cells in the gated small population which repels the DNA binding Hoechst 33342 dye (Fig. 2A). These drug efflux functions of SP cells are governed by the existence of multi-drug resistant ABC transporter protein ABCG2. We used verapamil which has an ability to suppress function of the transporter proteins, as a control. As a result, 
A
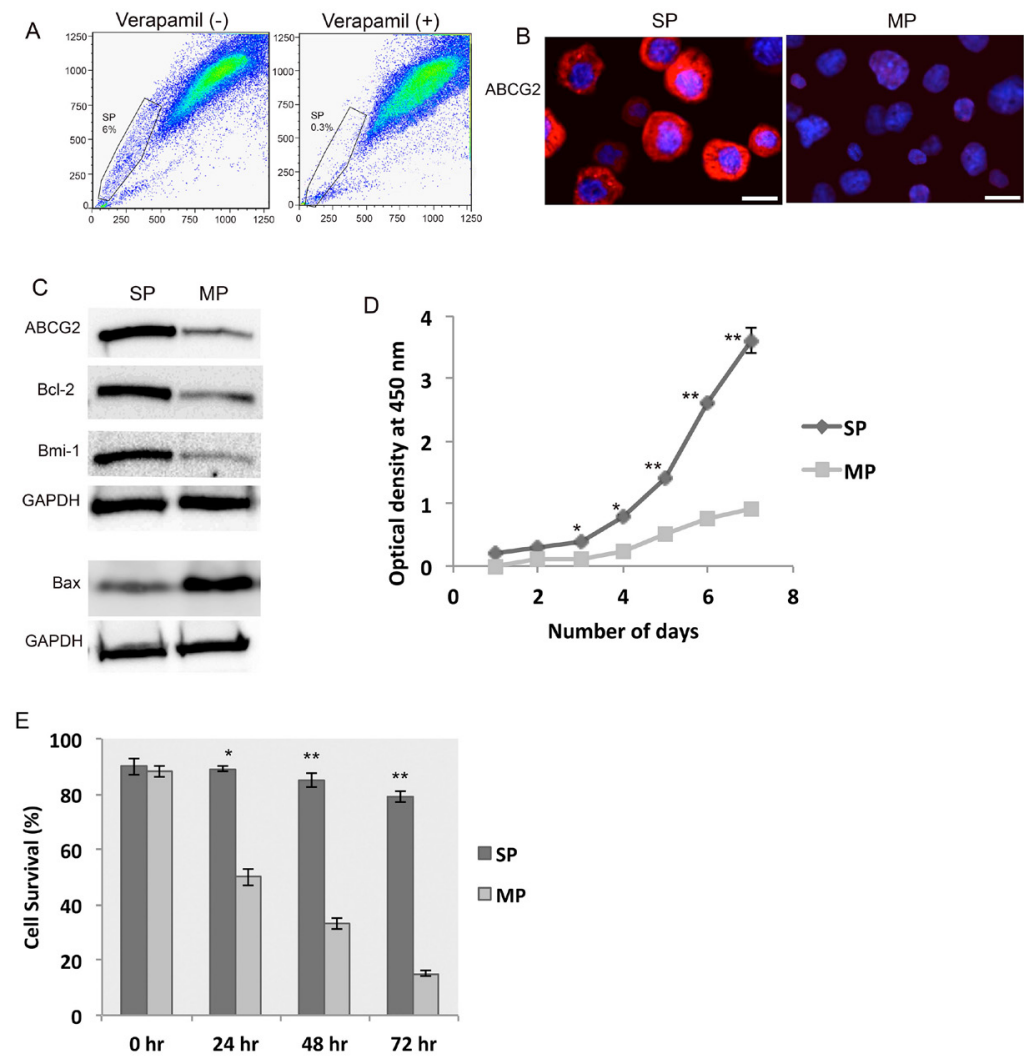

Figure 2. Characteristic features of Ovarian cancer SP cells.

(A) Fluorescence sorting of SP cells by the exclusion of Hoechst 33342 dye with or without verapamil. (B) Comparison of immunofluorescence staining pattern of $A B C$ transporter protein $A B C G 2$. (C) Western blot showing the protein expression levels of ABCG2 and apoptotic signaling proteins. (D) Measurement of growth rate of SP and MP cells. (D, E) Resistance of SP cells to 5-FU by chemoresistance assay. Scale bar represents $10 \mu \mathrm{M}$. Error bar: Standard deviation. ${ }^{*} P<0.05 ;{ }^{* *} P<0.01$.
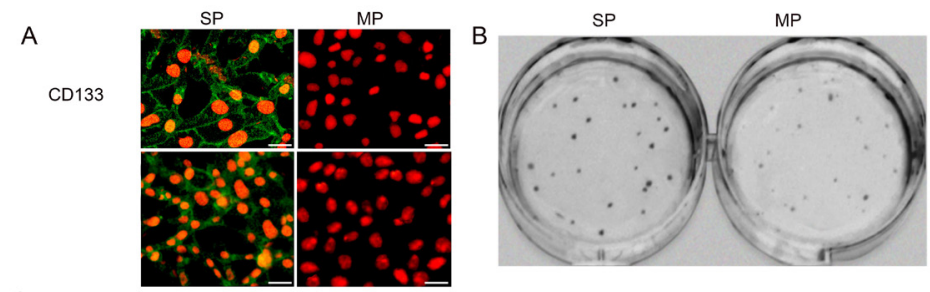

C
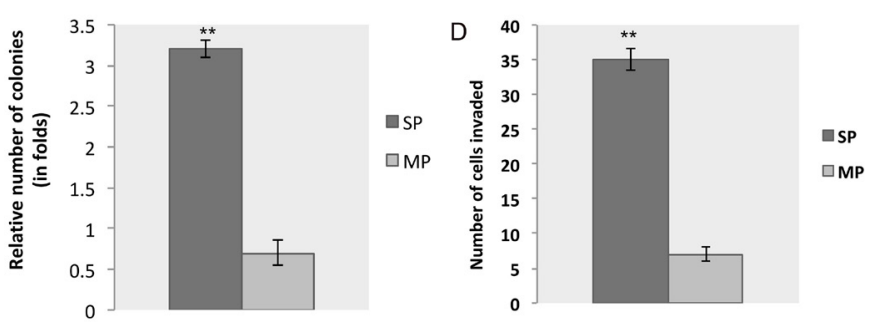

Figure 3. SP cell are highly tumorigenic.

(A) Immunofluorescence staining of SP showing expression of distinct surface antigens. (B) Efficiency of clone formation on soft agar plate and the quantitative graph (C) demonstrating the number of clones formed by SP and MP cells. (D) Representative graph made from the average values of matrigel invasion assay. Scale bar represents $11 \mu \mathrm{M}$. Error bar: Standard deviation; ${ }^{* * P} P<0.01$.

the SP cell population was significantly abolished (it became $0.3 \%$ ) when cells were incubated together with Hoechst 33342 and verapamil (Fig. 2A). Next, we have examined the sorted SP and MP cells for the expression of ABCG2 and apoptotic signaling proteins. By both, the immunocytochemistry and western blot, we found significantly enhanced ABCG2 protein levels in SP cells (Fig. $2 \mathrm{~B}$ and 2C). Similarly, the expression of anti-apoptotic protein $\mathrm{Bcl}-2$ and apoptotic signaling protein $\mathrm{Bax}$ were up- and down-regulated in SP cells, respectively (Fig. 2C). We also observed elevated expression of Bmi-1 is SP cells when compared to MP cells (Fig. 2C), which might collectively contribute to the accelerated growth of SP cells (Fig. 2D). Finally, we have performed apoptosis assessment of SP cells, by incubating SP cells with DNA targeting drug 5-FU. The cell viability measurement at differ- 

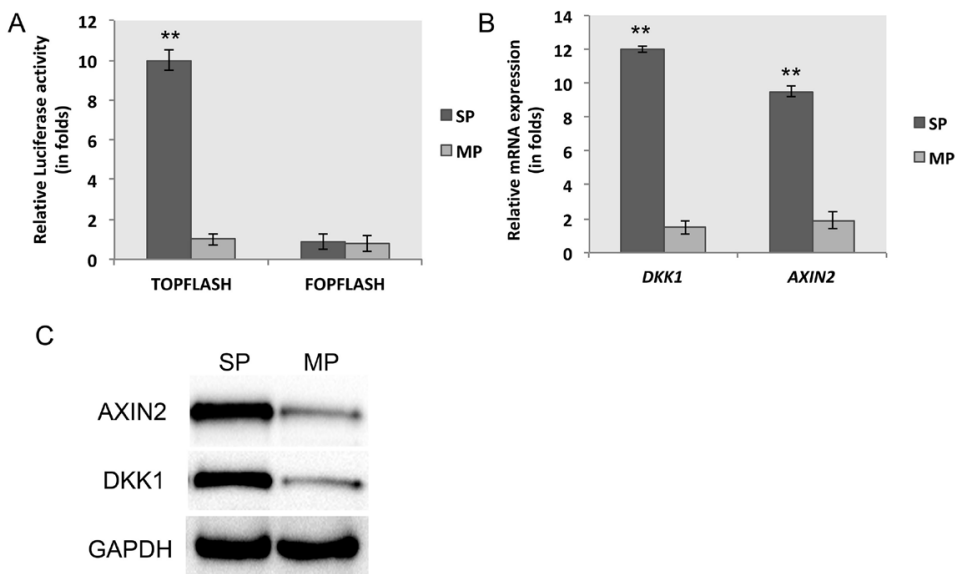

Figure 4. Aberrant activation of Wnt/B-catenin pathway in SP cells.

TOPFLASH luciferase activity (A), RT-PCR (B) and western blot analysis (C) determined the activation of Wnt/ $\beta$-catenin and evaluation of Wnt/ $\beta$-catenin downstream targets, respectively. Error bar: Standard deviation; ${ }^{* *} P<0.01$.

ent incubation time revealed that SP cells are assertively resistant to 5-FU and they grow normally up to 72 hours (Fig. 2E). However, in response to 5-FU the viability of MP started to gradually decline and ceased at a certain point. Therefore, all of these data confirm the phenotype of SP cells and their role in enhanced growth rate and chemoresistance owing to apoptosis attenuation.

\section{Immuno-positivity to CD133, CD117 and high tumorigenesis}

We have performed immunofluorescence assays to examine the expression pattern of stem cell marker proteins CD133 and CD177. We observed that ovarian cancer SP cells showed high positive staining towards CD133 and CD117. In contrast, the MP cells showed very mild positivity (Fig. 3A). Studies have shown that high expression profile of stemness genes/proteins is crucial for the selfrenewal, tumor-initiating and invasion. Consequently, the SP cells are highly capable of producing more colonies (3fold more) on the soft agar plate (Fig. 3B and 3C) and the number of SP cells invading through the matrigel (5-fold more) is drastically higher than MP cells (Fig. 3D). Therefore, the clonogenic and invasion potential of SP cells might be governed by stem like properties of SP cells.

\section{Aberrant regulation of the $W n t / \beta$-catenin pathway in SP cells}

We have analysed the activation of Wnt signaling pathway in SP and MP cells by TOPFLASH luciferase reporter assay. Here, the binding site of $\beta$-catenin is associated with TOPFLASH reporter whose activation is directly related to the activation of $\mathrm{Wnt} / \beta$-catenin, whereas the FOPFLASH reporter contains binding sites of mutated $\beta$-catenin, used as a negative control. From this assay, we found that the Wnt/ $\beta$-catenin transcriptional activity is significantly more elevated in SP than MP cells (Fig. 4A). Consequently, the relative $\mathrm{mRNA}$ and protein expression of Wnt/ $\beta$-catenin downstream targets, such as DKK1 and AXIN2, is significantly increased in SP cells (Fig. 4B and 4C).

\section{DISCUSSION}

Among EOC, Type II high grade serous ovarian cancer rapidly progresses, is highly aggressive and becomes lethal due to chemoresistance of CSCs (Abubaker et al., 2013). The existence of undifferentiated cancer stem cells (CSCs) or cancer initiating cells (CICs) inside the heterogeneous tumor can efficiently resist conventional treatment methods and have an ability to initiate tumor development and progression (Burkert et al., 2006; Dalerba et al., 2007). Side population cells are the minor, rare group of cancer stem-like progenitors involved in ejection of the Hoechst 33342 dye and exhibit characteristic features of CSCs, such as differential expression pattern of stem cell markers, chemo resistance, self-renewal, differentiation and tumor invasion (Haraguchi et al., 2006). These types of cells are present in tumor samples and various cell lines derived from different types of cancer. Therefore, as per the CSC theory, extermination of the CSCs cells is the ultimate goal for achieving better treatment strategy.

In high grade serous ovarian carcinoma, SP cells were identified by Hoechst 33342 efflux and further functional characterization revealed higher expression of the transporter protein ABCG2 in SP cells. Reports on many cancers have proven that presence of ABCG2 is the cause for SP cells to acquire the drug efflux phenotype and become resistant to the anti-cancer drugs. This was further confirmed by prolonged resistance and increased viability of ovarian cancer SP cells exposed to 5-FU even for 72 hours, and by that time the parental main population cells were deceased. In addition to 5-FU, we have also made an attempt to evaluate resistance of SP cells to docetaxel at different time points. Still, we were able to find susceptibility of SP cells to docetaxel treatment after 48 hours only. However, we are still standardizing the lethal effect of docetaxel in a dose dependent manner for our future experiments. In the meanwhile, it was demonstrated that transfection of ABCG2 over expression cassette into the main population cells can partially convert them to SP cells to acquire the same phenotype (Kopper, 2004; Bunting, 2006; Song et al., 2010). Therefore, CSCs are the major source for tumor metastasis and invasion (Song et al., 2010; He et al., 2014). By in vitro analysis, we found that SP cells are able to reproduce colonies faster and invaded the matrigel by more than three-folds. Therefore, SP cells are the key components for ovarian cancer metastasis and invasion.

Further, we have determined enrichment of surface antigens of stem cell, such as CD133 and CD117 in the 
small population of SP cells. We found differences in expression pattern of CSCs reporter proteins in SP and MP cells. The sorted SP cells showed positive and intense staining to CD133 and CD117 and their staining patterns on SP cells' surface are comparatively higher than MP cells. CD133 is a $120-\mathrm{kDa}$ membrane glycoprotein, previously identified in hematopoietic CSCs and ovary cancer SP cells (Yin et al., 1997; Bhatia, 2001). CD133+ cells from ovarian cancer demonstrated high potential of cell proliferation, clonogenic efficiency, therapy resistance and ability to form aggressive tumor rapidly in xenografts experiments (Baba et al., 2009). CD117 is a transmembrane, tyrosine kinase growth factor receptor, whose elevated expression was found in different types of tumors, and it has a functional role in cancer progression and invasion (Schmandt et al., 2003). Higher expres-

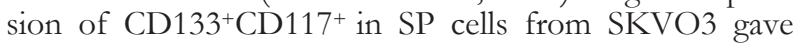
rise to aggressive tumor formation, when compared to wild-type cells (Ma et al., 2010). Therefore, the SP cells that express these cell surface antigens are also able to grow and differentiate rapidly when compared to non-SP cells. Therefore, we also analyzed the stem cell surface markers which are primarily contributed to self-renewal and tumorigenesis, and we found enhanced expression of CD133 and CD117 in ovary cancer SP cells. These data fit with the previous findings in several cancers, such as the lung, colon, oral, ovary cancers etc., which suggests an increased proliferation, differentiation, self-renewal and tumor initiating potential of SP cells (Mărgăritescu et al., 2011; Xie et al., 2014; Zhang et al., 2015; Miyata et al., 2017). In future research, RT-PCR will be conducted to evaluate the wide range of stem cell marker genes in ovary cancer samples and in its cell lines as well.

We observed that Bmi-1, CD133 and CD117 displayed high expression profile in SP cells. Bmi-1 belongs to the family of Polycomb group (PcG) shown to be involved in chemo resistance and maintenance of self-renewal properties of CSCs. Bmi-1 knock down increases CSCs' sensitivity towards anti-cancer drugs and induces apoptosis (Lessard et al., 2003). Consequently, the SP cells are proliferating much faster than MP cells and this explains the nature of rapid cancer cell proliferation. Further, the rate of apoptosis was dramatically reduced in SP cells by the evident protein up-regulation, Bcl-2 and suppression of the Bax protein.

The Notch and Wnt signaling pathway are influenced by BMI-1 gene and the major function of BMI1 was suggested to be involved in stem cell proliferation of mammals (Liu et al., 2006). The evolutionarily conserved Wnt/ $\beta$-catenin signaling pathway regulates genes involved in cell proliferation, migration and invasion (Cheng et al., 2019), whose activation and stemness function was first identified in the ovarian cancer (Clevers et al., 2006; van Amerongen et al., 2009). In view of this, we initially targeted Wnt/ $\beta$-catenin signaling pathway in ovary cancer SP cells. Interestingly, our data revealed that relative mRNA level of $\mathrm{Wnt} / \beta$-catenin targets (DKK1, AXIN2) are accelerated more in SP cells and thus confirms hyperactivation of Wnt/ $\beta$-catenin signaling pathways in SP cells. Similar to our findings, increased Wnt/ $\beta$-catenin was found in HNSCC and the authored claimed that this abnormal activation promotes CSCs mediated tumorigenesis and invasion. Our further research will be involving RNAi approach for Wnt/ $\beta$ catenin downstream targets, such as DKK1 and AXIN2. As a preliminary approach, we have made RNAi depletion of DKK1 in SP cells and our data revealed that DKK1 depleted SP cells significantly decrease the tumor initiation and cell invasion potential of ovarian cancer SP cells (Supplementary Fig. 1 at https://ojs.ptbioch.edu.pl/ index.php/abp/). Therefore, SP cells already resist DNA targeting drugs and escapes apoptosis, and in addition enhanced expression of DKK1 and AXIN2 collectively paves the way for rapid cancer progression, metastasis and invasion. Further, our supplementary data suggest that aberrant expression of Wnt/ $\beta$-catenin targets, such as DKK1, poses a promising role in cancer progression and invasion. However, lack of animal models, other in vivo experiments and establishment of cell lines which exert complete knock down of DKK1 and AXIN2 are the major limitations from our present study and the previous reports as well (Yang et al., 2006; Song et al., 2010). Keeping this in mind, we are planning to execute more in vitro and in vivo experiments in the future to elucidate the molecular mechanism and carcinogenesis behind the aberrant activation of Wnt/ $\beta$-catenin signaling and its downstream targets in CSCs. Taken together, our data clearly displayed that assorted SP cells are found to be more tumorigenic than the main population, as they showed high efficiency of clone formation, more defense against 5-FU even at the extended incubation time, and were aggressively invading in the matrigel invasion assay. Studies focusing on elucidating the comprehensive mechanism and factors triggering Wnt/ $\beta$-catenin pathways, resistance to DNA binding drugs, self-renewal ability and carcinogenesis would be definitely beneficial and afford better treatment approach in order to achieve disease free goal.

\section{Conflict of Interest}

All authors who contributed to this manuscript declare no conflict of interest and they all agree with the content of this manuscript.

\section{REFERENCES}

Abubaker K, Latifi A, Luwor R, Nazaretian S, Zhu H, Quinn MA, Thompson EW, Findlay JK, Ahmed N (2013) Short-term single treatment of chemotherapy results in the enrichment of ovarian cancer stem cell-like cells leading to an increased tumor burden. Mol Cancer 12: 24. https://doi.org/10.1186/1476-4598-12-24

Baba T, Convery PA, Matsumura N, Whitaker RS, Kondoh E, Perry T, Huang Z, Bentley RC, Mori S, Fujii S, Marks JR (2009) Epigenetic regulation of CD133 and tumorigenicity of CD133f ovarian cancer cells. Oncogene 28: 209-218. https://doi.org/10.1038/onc.2008.374

Baccelli I, Trumpp A (2012) The evolving concept of cancer and metastasis stem cells. J Cell Biol 198: 281-293. https://doi.org/10.1083/ jcb.201202014

Bhatia M (2001) AC133 expression in human stem cells. Leukemia 15: 1685-1688. https://doi.org/10.1038/sj.leu.2402255

Bonnet D, Dick JE (1997) Human acute myeloid leukemia is organized as a hierarchy that originates from a primitive hematopoietic cell. Nat Med 3: 730-737. https://doi.org/10.1038/nm0797-730

Bunting KD (2002) ABC transporters as phenotypic markers and functional regulators of stem cells. Stem Cells 20: 11-20. https://doi. org/10.1038/nm0797-73010.1002/stem.200011

Burkert J, Wright NA, Alison MR (2006) Stem cells and cancer: an intimate relationship. J Pathol 209: 287-97. https://doi.org/10.1002/ path.2016

Cheng X, Xu X, Chen D, Zhao F, Wang W (2019) Therapeutic potential of targeting the $\mathrm{Wnt} / \beta$-catenin signaling pathway in colorectal cancer. Biomed Pharmacother 110: 473-481. https://doi.org/10.1016/j. biopha.2018.11.082

Clevers H (2006) Wnt/beta-catenin signaling in development and disease. Cell 127: 469-480. https://doi.org/10.1016/j.cell.2006.10.018

Clevers H (2011) The cancer stem cell: premises, promises and challenges. Nat Med 17: 313-319. https://doi.org/10.1038/nm.2304

Dalerba P, Clarke MF (2007) Cancer stem cells and tumor metastasis: first steps into uncharted territory. Cell Stem Cell 1: 241-242. https://doi.org/10.1016/j.stem.2007.08.012

Dalerba P, Dylla SJ, Park IK, Liu R, Wang X, Cho RW, Hoey T, Gurney A, Huang EH, Simeone DM, Shelton AA (2007) Phenotypic characterization of human colorectal cancer stem cells. Proc Natl Acad Sci U S A 104: 10158-10163. https://doi.org/10.1073/ pnas.0703478104 
Dean M, Fojo T, Bates S (2005) Tumour stem cells and drug resistance. Nat Rev Cancer 5: 275-284. https://doi.org/10.1038/nrc1590

Haraguchi N, Inoue H, Tanaka F, Mimori K, Utsunomiya T, Sasaki A, Mori M (2006) Cancer stem cells in human gastrointestinal cancers. Human Cell 19: 24-29. https://doi.org/10.1111/j.1749-0774

He QZ, Luo XZ, Wang K, Zhou Q, Ao H, Yang Y, Li SX, Li Y, Zhu HT, Duan T (2014) Isolation and characterization of cancer stem cells from high-grade serous ovarian carcinomas. Cell Physiol Biochem 33: 173-184. https://doi.org/10.1159/000356660

Hill RP (2006) Identifying cancer stem cells in solid tumors: case not proven. Cancer Res 66: 1891-1895. https://doi.org/10.1158/00085472.CAN-05-3450

Hill RP, Perris R (2006) "Destemming" cancer stem cells. J Natl Cancer Inst 99: 1435-1440. https://doi.org/10.1093/jnci/djm136

Ho MM, Ng AV, Lam S, Hung JY (2007) Side population in human lung cancer cell lines and tumors is enriched with stem-like cancer cells. Cancer Res 67: 4827-4833. https://doi.org/10.1158/0008-5472

Kopper L, Hajdu M (2004) Tumor stem cells. Pathol Oncol Res 10: 69_ 73. https://doi.org/10.1007/BF02893458

Kurman RJ, Shih I (2010) The origin and pathogenesis of epithelial ovarian cancer: a proposed unifying theory. Am J Surg Pathol 34: 433-443. https://doi.org/10.1097/PAS.0b013e3181cf3d79

Kurman RJ, Shih I (2011) Molecular pathogenesis and extraovarian origin of epithelial ovarian cancer shifting the paradigm. Hum Pathol 42: 918-931. https://doi.org/10.1016/j.humpath.2011.03.003

Liu S, Dontu G, Mantle ID, Patel S, Ahn NS, Jackson KW, Suri P, Wicha MS (2006) Hedgehog signaling and Bmi-1 regulate self-renewal of normal and malignant human mammary stem cells. Cancer Res 66: 6063-6071. https://doi.org/10.1158/0008-5472.CAN-060054

Lengyel E (2010) Ovarian cancer development and metastasis. Am J Pathol 177: 1053-1064. https://doi.org/10.2353/aipath.2010.100105

Lessard J, Sauvageau G (2003) Bmi-1 determines the proliferative capacity of normal and leukaemic stem cells. Nature 423: 255-260. https://doi.org/10.1038/nature01572

Ma L, Lai D, Liu T, Cheng W, Guo L (2010) Cancer stem-like cells can be isolated with drug selection in human ovarian cancer cell line SKOV3. Acta Biochim Biophys Sin 42: 593-602. https://doi. org/10.1093/abbs/gmq067

Mărgăritescu C, Pirici D, Simionescu CR, Stepan A (2011) The utility of CD44, CD117 and CD133 in identification of cancer stem cells (CSC) in oral squamous cell carcinomas (OSCC). Rom J Morphol Embryol 52: 985-983

Masison C, Brady J, Hao K, Berkowitz RS, Mok S (2006) Expression profiling of mucinous tumors of the ovary identifies genes of clinicopathologic importance. Clin Cancer Res 12: 690-700. https://doi. org/10.1158/1078-0432.CCR-05-1110

Miyata T, Oyama T, Yoshimatsu T, Higa H, Kawano D, Sekimura A, Yamashita N, So T, Gotoh A (2017) The clinical significance of cancer stem cell markers ALDH1A1 and CD133 in lung adenocarcinoma. Anticancer Res 37: 2541-2547. https://doi.org/10.21873/anticanres. 11597

Schmandt RE, Broaddus R, Lu KH, Shvartsman H, Thornton A, Malpica A, Sun C, Bodurka DC, Gershenson DM (2003) Expression of c-ABL, c-Kit and platelet-derived growth factor receptor-beta in ovarian serous carcinoma and normal ovarian surface epithelium. Cancer 98: 758-764. https://doi.org/10.1002/cncr.11561

Song J, Chang I, Chen Z, Kang M, Wang CY (2010) Characterization of side populations in HNSCC: highly invasive, chemoresistant and abnormal wnt signaling. PLoS One 5: e11456. https://doi. org/10.1371/journal.pone.0011456

Taciak B, Pruszynska I, Kiraga L, Bialasek M, Krol M (2018) Wnt signaling pathway in development and cancer. I Physiol Pharmacol 69: 185-196. https://doi.org/10.26402/jpp.2018.2.07

Vathipadiekal V, Saxena D, Mok SC, Hauschka PV, Ozbun L, Birrer MJ (2012) Identification of a potential ovarian cancer stem cell gene expression profile from advanced stage papillary serous ovarian cancer. PLoS One 7: e29079. https://doi.org/10.1371/journal. pone.0029079

van Amerongen R, Nusse R (2009) Towards an integrated view of Wnt signaling in development. Dev Camb Engl 136: 3205-3214. https:// doi.org/10.1242/dev.033910

Visvader JE, Lindeman GJ (2008) Cancer stem cells in solid tumours: accumulating evidence and unresolved questions. Nat Rev Cancer 8: 755-768. https://doi.org/10.1038/nrc2499

Wamunyokoli FW, Bonome T, Lee JY, Feltmate CM, Welch WR, Radonovich M, Pise-Masison C, Brady J, Hao K, Berkowitz RS, Mok S (2006) Expression profiling of mucinous tumors of the ovary identifies genes of clinicopathologic importance. Clin Cancer Res 12: 690-700. https://doi.org/10.1158/1078-0432.CCR-05-1110

Xie ZY, Lv K, Xiong Y, Guo WH (2014) ABCG2-meditated multidrug resistance and tumor-initiating capacity of side population cells from colon cancer. Oncol Res Treat 37: 666-672. https://doi. org/10.1159/000368842

Yang F, Zeng Q, Yu G, Li S, Wang CY (2006) Wnt/ $\beta$-catenin signaling inhibits death receptor-mediated apoptosis and promotes invasive growth of HNSCC. Cell Signal 18: 679-687. https://doi. org/10.1016/j.cellsig.2005.06.015

Yin AH, Miraglia S, Zanjani ED, Almeida-Porada G, Ogawa M, Leary AG, Olweus J, Kearney J, Buck DW (1997) AC133, a novel marker for human hematopoietic stem and progenitor cells. Blood 90: 50025012

Zhang QH, Dou HT, Xu P, Zhuang SC, Liu PS (2015) Tumor recurrence and drug resistance properties of side population cells in high grade ovary cancer. Drug Res 65: 153-137. https://doi. org/10.1055/s-0034-1375609 